\title{
Factorization in spin systems under general fields and separable ground-state engineering
}

\author{
M. Cerezo, R. Rossignoli, and N. Canosa \\ Instituto de Física de La Plata and Departamento de Física, Universidad Nacional de La Plata, C.C.67, La Plata 1900, Argentina
}

(Received 23 June 2016; published 24 October 2016)

\begin{abstract}
We discuss ground-state factorization schemes in spin $S$ arrays with general quadratic couplings under general magnetic fields, not necessarily uniform or transverse. It is shown that, given arbitrary spin alignment directions at each site, nonzero $X Y Z$ couplings between any pair and fields at each site always exist such that the ensuing Hamiltonian has an exactly separable eigenstate with the spins pointing along the specified directions. Furthermore, by suitable tuning of the fields this eigenstate can always be cooled down to a nondegenerate ground state. It is also shown that in open one-dimensional systems with fixed arbitrary first-neighbor couplings at least one separable eigenstate compatible with an arbitrarily chosen spin direction at one site is always feasible if the fields at each site can be tuned. We demonstrate as well that in the vicinity of factorization, i.e., for small perturbations in the fields or couplings, pairwise entanglement reaches full range. Some noticeable examples of factorized eigenstates are unveiled. The present results open the way for separable ground-state engineering. A notation to quantify the complexity of a given type of solution according to the required control on the system couplings and fields is introduced.
\end{abstract}

DOI: 10.1103/PhysRevA.94.042335

\section{INTRODUCTION}

Over the last two decades quantum information and quantum computation sciences have experienced an extraordinary theoretical and experimental progress [1-3]. In particular, the possibility of performing quantum information processing and quantum simulation tasks in archetypal many-body systems such as spin arrays has attracted considerable attention [4-11]. Furthermore, the recent noticeable advances in control techniques of quantum systems have made it possible to engineer and simulate spin interactions by means of cold atoms in optical lattices [12-14], superconducting Josephson junctions [15-18], or trapped ions [14,19-24], leading to an ever increasing interest in these strongly correlated systems.

In this framework, it is well known that the exact eigenstates of interacting spin systems in the presence of an external magnetic field are typically entangled states. However, one of the most exceptional features of these systems is that under certain conditions they can possess a completely separable ground state (GS), i.e., a factorized GS which can be expressed as the product of single spin states [25]. The remarkable phenomenon of factorization has been thoroughly studied in spin systems immersed in a uniform transverse field [26-35], and in finite anisotropic $X Y Z$ spin chains the transverse factorizing field has been shown [31,32] to correspond to a GS $S_{z}$ parity transition, the ensuing separable GS being twofold degenerate. Recently, in Ref. [36] we studied $X Y Z$ models with uniform nontransverse fields, where it was shown that a uniform, maximally aligned, nondegenerate, completely separable GS can exist in both ferromagnetic- and antiferromagnetic-type systems for fields parallel to a principal plane of the coupling.

In this work we show that if some control over the couplings or the fields is feasible, then completely separable exact ground states can be engineered in general $X Y Z$-type systems. This point is important for the first basic step in most quantum information processes [37] and quantum simulation schemes [14,24], since they are based on an initial fully separable state of the qubits, assumed to be reached with high fidelity.
Whenever such initial state is only approximately achieved or is prone to decoherence, additional error correction must be implemented [38]. Therefore, the possibility of having an exactly separable GS at finite magnetic fields even in the presence of strong interactions between the spins is highly desirable, especially if such GS is nondegenerate and can be well separated from the remaining spectrum. Such possibility can be also useful in schemes for quantum annealing $[39,40]$.

We first show that for arbitrary alignment directions at each site, compatible nonzero $X Y Z$ couplings between any pair of spins and concomitant finite factorizing fields always exist such that the separable state is an exact eigenstate of the system. Moreover, such state can always be made a nondegenerate well-separated GS by appropriately tuning the fields. In addition, it is shown, remarkably, that for an arbitrary quadratic coupling between two spins and an arbitrarily chosen spin alignment direction of one of the spins, there is always an alignment direction of the remaining spin compatible with an exactly separable eigenstate. This result enables us to engineer separable GSs in systems with arbitrary first-neighbor couplings at least in one-dimensional-type geometries, if fields can be tuned. Furthermore, the factorizing fields for a single pair can be always chosen as uniform, though in general nontransverse. A complexity classification scheme for the control required on the couplings and fields is accordingly introduced. This general framework also allows us to identify and prove the existence of nontrivial separable eigenstates for certain couplings, fields, and geometries, like the spinspiral-type solution which will be discussed. We also suggest two experimental implementations for which the proposed methods could be realized. A final but not less important aspect is that the present general factorization points, arising for not-necessarily uniform couplings and nontransverse fields, can also be associated to an entanglement transition: pairwise entanglement, though obviously vanishing at factorization, will be shown to reach full range in its vicinity if either the fields or couplings are perturbed, in agreement with previous results for uniform fields $[28,31,36]$. 
The general rigorous results are presented and demonstrated in Sec. II and the Appendix. Special examples of factorized eigenstates are discussed in Sec. III. The ensuing GS engineering schemes, complexity classification, and experimental implementation are discussed in Sec. IV. Conclusions are finally drawn in Sec. V.

\section{EXACTLY SEPARABLE EIGENSTATES}

\section{A. Separability conditions for general quadratic couplings}

We consider an array of $N$ spins $S_{i}$, not necessarily equal, interacting through general quadratic couplings of arbitrary range in the presence of a general magnetic field $\boldsymbol{h}^{i}=\left(h_{x}^{i}, h_{y}^{i}, h_{z}^{i}\right)$. The Hamiltonian is

$$
\begin{aligned}
H & =-\sum_{i} \boldsymbol{h}^{i} \cdot \boldsymbol{S}_{i}-\frac{1}{2} \sum_{i \neq j} \boldsymbol{S}_{i} \cdot \mathcal{J}^{i j} \boldsymbol{S}_{j} \\
& =-\sum_{i, \mu} h_{\mu}^{i} S_{i}^{\mu}-\frac{1}{2} \sum_{i \neq j, \mu, \nu} J_{\mu \nu}^{i j} S_{i}^{\mu} S_{j}^{\nu},
\end{aligned}
$$

where $i, j$ label the sites in the array; $S_{i}^{\mu}, \mu=x, y, z$ denote the spin components at site $i$; and $J_{\mu \nu}^{i j}=J_{\nu \mu}^{j i}$ are the coupling strengths between spins at sites $i$ and $j$, with $\mathcal{J}^{i j}$ a matrix of elements $J_{\mu \nu}^{i j}$. The $X Y Z$ case corresponds to $\mathcal{J}^{i j}$ diagonal $\forall i, j$ $\left(J_{\mu \nu}^{i j}=\delta_{\mu \nu} J_{\mu}^{i j}\right)$. The Hamiltonian (1) will possess a completely separable eigenstate of the form

$$
|\Theta\rangle=\otimes_{i=1}^{n} R_{i}\left|0_{i}\right\rangle=|\nearrow \rightarrow \swarrow \nwarrow \ldots\rangle, \quad R_{i}=e^{-l \phi_{i} S_{i}^{z}} e^{-l \theta_{i} S_{i}^{y}},
$$

where $\left|0_{i}\right\rangle$ is the local state with maximum spin along the $z$ axis $\left(S_{i}^{z}\left|0_{i}\right\rangle=S_{i}\left|0_{i}\right\rangle\right)$ and $R_{i}$ is a rotation such that the resulting spin alignment direction is $\boldsymbol{n}_{i}=$ $\left(\sin \theta_{i} \cos \phi_{i}, \sin \theta_{i} \sin \phi_{i}, \cos \theta_{i}\right)$, if two sets of conditions are met [36]. The first ones are the pairwise field independent equations which relate the alignment directions with the exchange couplings:

$$
\boldsymbol{n}_{i}^{x^{\prime}} \cdot \mathcal{J}^{i j} \boldsymbol{n}_{j}^{x^{\prime}}=\boldsymbol{n}_{i}^{y^{\prime}} \cdot \mathcal{J}^{i j} \boldsymbol{n}_{j}^{y^{\prime}}, \quad \boldsymbol{n}_{i}^{x^{\prime}} \cdot \mathcal{J}^{i j} \boldsymbol{n}_{j}^{y^{\prime}}=-\boldsymbol{n}_{i}^{y^{\prime}} \cdot \mathcal{J}^{i j} \boldsymbol{n}_{j}^{x^{\prime}} .
$$

Here $\boldsymbol{n}_{i}^{x^{\prime}}=\left(\cos \theta_{i} \cos \phi_{i}, \cos \theta_{i} \sin \phi_{i},-\sin \theta_{i}\right), \boldsymbol{n}_{i}^{y^{\prime}}=\left(-\sin \phi_{i}\right.$, $\left.\cos \phi_{i}, 0\right)$ are the corresponding rotated vectors orthogonal to $\boldsymbol{n}_{i}^{z^{\prime}}=\boldsymbol{n}_{i}$, such that $\left(\boldsymbol{n}_{i}^{x^{\prime}}, \boldsymbol{n}_{i}^{y^{\prime}}, \boldsymbol{n}_{i}\right)$ forms an orthonormal triad. Equations (4) mean that the strengths $J_{\mu^{\prime} \nu^{\prime}}^{i j}=\boldsymbol{n}_{i}^{\mu^{\prime}} \cdot \mathcal{J}^{i j} \boldsymbol{n}_{j}^{\nu^{\prime}}$ associated with the rotated spin operators $S_{i}^{\mu^{\prime}}=R_{i} S_{i}^{\mu} R_{i}^{\dagger}\left(S_{i}\right.$. $\left.\mathcal{J}^{i j} \boldsymbol{S}_{j}=\sum_{\mu, \nu} J_{\mu^{\prime} \nu^{\prime}}^{i j} S_{i}^{\mu^{\prime}} S_{j}^{\nu^{\prime}}\right)$ satisfy $J_{x^{\prime} x^{\prime}}^{i j}=J_{y^{\prime} y^{\prime}}^{i j}$ and $J_{x^{\prime} y^{\prime}}^{i j}=$ $-J_{y^{\prime} x^{\prime}}^{i j}$, ensuring that $H$ does not connect $|\Theta\rangle$ with two spin excitations.

The second set are the local field dependent equations which determine the factorizing fields $\boldsymbol{h}^{i}$ at each site:

$$
\boldsymbol{h}^{i}=\boldsymbol{h}_{\|}^{i}+\boldsymbol{h}_{\perp}^{i},
$$

where $\boldsymbol{h}_{\|}^{i}=h_{\|}^{i} \boldsymbol{n}_{i}$ is an arbitrary field parallel to the local spin alignment direction $\boldsymbol{n}_{i}$ and

$$
\boldsymbol{h}_{\perp}^{i}=\boldsymbol{n}_{i} \times\left(\boldsymbol{n}_{i} \times\left(\sum_{j} S_{j} \mathcal{J}^{i j} \boldsymbol{n}_{j}\right)\right),
$$

is a field orthogonal to the local alignment direction $\boldsymbol{n}_{i}$, which represents the nontransverse factorizing field of lowest magnitude and ensures that $H$ will not connect $|\Theta\rangle$ with single spin excitations: Eq. (6), equivalent to $\boldsymbol{h}_{\perp}^{i}=\sum_{j} \boldsymbol{h}_{\perp}^{i j}$ with $\boldsymbol{h}_{\perp}^{i j}=$ $-S_{j}\left[\mathcal{J}^{i j} \boldsymbol{n}_{j}-\boldsymbol{n}_{i}\left(\boldsymbol{n}_{i} \cdot \mathcal{J}^{i j} \boldsymbol{n}_{j}\right)\right]$, implies $h_{\mu^{\prime}}^{i}=-\sum_{j} S_{j} J_{\mu^{\prime} z^{\prime}}^{i j}$ for $\mu^{\prime}=x^{\prime}, y^{\prime}$.

If Eqs. (4)-(6) are fulfilled, then $H|\Theta\rangle=E_{\Theta}|\Theta\rangle$, with

$$
\begin{aligned}
E_{\Theta} & =-\sum_{i}\left\langle\boldsymbol{S}_{i}\right\rangle \cdot \boldsymbol{h}^{i}-\frac{1}{2} \sum_{i \neq j}\left\langle\boldsymbol{S}_{i}\right\rangle \cdot \mathcal{J}^{i j}\left\langle\boldsymbol{S}_{j}\right\rangle \\
& =-\sum_{i} S_{i} h_{\|}^{i}-\frac{1}{2} \sum_{j \neq i, \mu, \nu} S_{i} S_{j} J_{\mu \nu}^{i j} n_{i \mu} n_{j \nu},
\end{aligned}
$$

where $\left\langle\boldsymbol{S}_{i}\right\rangle \equiv\left\langle\Theta\left|\boldsymbol{S}_{i}\right| \Theta\right\rangle=S_{i} \boldsymbol{n}_{i}$. This energy is split into two contributions: the first one is associated with the parallel field components $\boldsymbol{h}_{\|}^{i}=h_{\|}^{i} \boldsymbol{n}_{i}$ and the second one, independent of $\boldsymbol{h}_{\|}^{i}$, with the couplings. The parallel components $\boldsymbol{h}_{\|}^{i}$ can then be used to shift the energy of the factorized state and hence to cool it down to a GS, as discussed below.

\section{B. Fundamental properties}

We now provide five fundamental properties of the previous maximally aligned separable eigenstates. For the sake of clarity proof details are presented in the Appendix.

Lemma 1. If Eqs. (4)-(6) are satisfied, the state $|\Theta\rangle$ given by Eq. (3) will always become a nondegenerate GS of $H$ for sufficiently strong yet finite parallel fields $\boldsymbol{h}_{\|}^{i}=h_{\|}^{i} \boldsymbol{n}_{i}$.

Proof. This result is apparent as no state $|\Psi\rangle$ orthogonal to $|\Theta\rangle$ will have an energy $\langle H\rangle_{\Psi} \equiv\langle\Psi|H| \Psi\rangle$ which decreases more rapidly with the applied fields $h_{\|}^{i}$ than $E_{\Theta}$, since $\langle\Theta| S_{i}$. $\boldsymbol{n}_{i}|\Theta\rangle=S_{i}$ is maximum. Hence, a finite threshold value $h_{\| c}$ will always exist such that $|\Theta\rangle$ becomes a nondegenerate GS if $h_{\|}^{i}>h_{\| c} \forall i$. Moreover, the energy gap with the first excited state can be made as large as desired by increasing the values of $h_{\|}^{i}$.

Lemma 2. Given two arbitrary alignment directions $\boldsymbol{n}_{i}, \boldsymbol{n}_{j}$ at sites $i, j$, a nonzero $X Y Z$-type coupling $J_{\mu \nu}^{i j}=J_{\mu}^{i j} \delta_{\mu \nu}$ always exists such that Eqs. (4) are fulfilled.

This lemma implies that for arbitrary alignment directions $\boldsymbol{n}_{i}$ at each site of the array, $X Y Z$ couplings $J_{\mu}^{i j}$ and suitable fields $\boldsymbol{h}^{i}$ always exist such that the associated factorized state $|\Theta\rangle$ is an exact GS of $H$.

Proof. For $J_{\mu \nu}^{i j}=J_{\mu}^{i j} \delta_{\mu \nu}$, Eqs. (4) can be conveniently rewritten in vector form as

$$
\boldsymbol{J}^{i j} \cdot \boldsymbol{U}^{i j}=0, \boldsymbol{J}^{i j} \cdot \boldsymbol{V}^{i j}=0,
$$

where $\boldsymbol{J}^{i j}=\left(J_{x}^{i j}, J_{y}^{i j}, J_{z}^{i j}\right)$ is the exchange coupling vector and $\boldsymbol{U}^{i j}, \boldsymbol{V}^{i j}$ are the vectors defined as $\boldsymbol{U}^{i j}=\boldsymbol{n}_{i}^{x^{\prime}} *$ $\boldsymbol{n}_{j}^{x^{\prime}}-\boldsymbol{n}_{i}^{y^{\prime}} * \boldsymbol{n}_{j}^{y^{\prime}}, \quad V^{i j}=\boldsymbol{n}_{i}^{x^{\prime}} * \boldsymbol{n}_{j}^{y^{\prime}}+\boldsymbol{n}_{i}^{y^{\prime}} * \boldsymbol{n}_{j}^{x^{\prime}}, \quad$ with $\boldsymbol{n} * \boldsymbol{m}=$ $\left(n_{x} m_{x}, n_{y} m_{y}, n_{z} m_{z}\right)$ the Hadamard product, such that $\boldsymbol{n}_{i}$. $\mathcal{J}^{i j} \boldsymbol{n}_{j}=\boldsymbol{J} \cdot\left(\boldsymbol{n}_{i} * \boldsymbol{n}_{j}\right)$ for $\mathcal{J}^{i j}$ diagonal. Hence, by choosing $\boldsymbol{J}^{i j}$ orthogonal to the subspace generated by $\boldsymbol{U}^{i j}$ and $\boldsymbol{V}^{i j}$, Eqs. (8) [and then Eq. (4)] are fulfilled. The fields can then be obtained from Eqs. (5)-(6). And by applying sufficiently strong parallel fields $\boldsymbol{h}_{\|}^{i},|\Theta\rangle$ can be made a GS (Lemma 1).

Note that two distinct situations are implied by Eq. (8), as depicted in Fig. 1: If $\boldsymbol{U}^{i j}$ and $\boldsymbol{V}^{i j}$ are linearly independent, it 

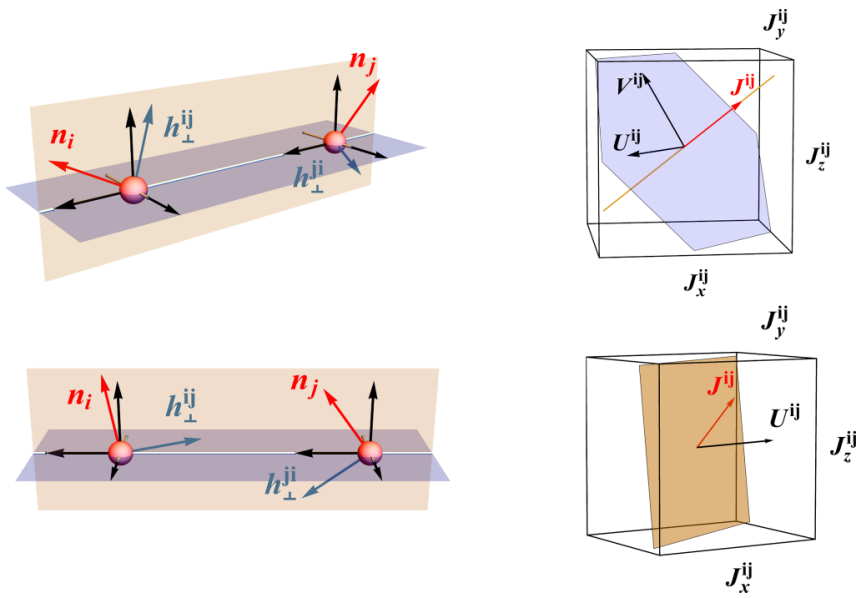

FIG. 1. Left column: Schematic representation of the spin alignment directions $\boldsymbol{n}_{i}$ and $\boldsymbol{n}_{j}$ and the perpendicular factorizing fields $\boldsymbol{h}_{\perp}^{i j}$ and $\boldsymbol{h}_{\perp}^{j i}$ [Eq. (6)]. Right column: Exchange couplings in the exchange couplings space compatible with the spin alignment configuration. Top row: $\boldsymbol{U}^{i j}$ and $\boldsymbol{V}^{i j}$ are linearly independent and Eqs. (8) define a line [Eq. (9)] of possible exchange couplings. Bottom row: $\boldsymbol{U}^{i j}$ and $\boldsymbol{V}^{i j}$ are linearly dependent (as $\boldsymbol{n}_{i}$ and $\boldsymbol{n}_{j}$ belong to a principal plane) and Eqs. (8) define a plane of possible exchange couplings [Eq. (A4)].

determines a line of compatible exchange vectors orthogonal to the plane generated by $\boldsymbol{U}^{i j}$ and $\boldsymbol{V}^{i j}$, i.e.,

$$
\boldsymbol{J}^{i j}=j^{i j}\left(\boldsymbol{U}^{i j} \times \boldsymbol{V}^{i j}\right),
$$

with $j^{i j}$ an arbitrary real constant.

On the other hand, if $\boldsymbol{U}^{i j}$ and $\boldsymbol{V}^{i j}$ are linearly dependent, it defines a plane of compatible exchange couplings. This case arises whenever (i) $\boldsymbol{n}_{i}$ and $\boldsymbol{n}_{j}$ belong to the same principal plane (i.e., $n_{j \sigma}=n_{i \sigma}=0$ for some $\sigma=z, x$ or $y$ ); (ii) $\boldsymbol{n}_{j}$ is the reflection of $\boldsymbol{n}_{i}$ with respect to a principal plane $\left(\left|n_{j \mu}\right|=\right.$ $\left|n_{i \mu}\right| \forall \mu$, with $n_{j \sigma}=-n_{i \sigma}$ for just one component $\sigma$ ); and (iii) $\boldsymbol{n}_{i}=-\boldsymbol{n}_{j}$, i.e., antiparallel alignment directions. In these cases $\boldsymbol{V}^{i j}$ vanishes for the present choice of orthogonal vectors $\boldsymbol{n}_{i}^{\mu^{\prime}}$ and the plane of compatible exchange couplings is that orthogonal to $\boldsymbol{U}^{i j}$. The explicit expressions for $\boldsymbol{J}^{i j}$ are given in the Appendix.

Lemma 3. Given an arbitrary quadratic coupling $\boldsymbol{S}_{i} \mathcal{J}^{i j} \boldsymbol{S}_{j}=$ $\sum_{\mu, v} J_{\mu \nu}^{i j} S_{i}^{\mu} S_{j}^{\nu}$ between two spins and an arbitrary alignment direction $\boldsymbol{n}_{j}$ of one of the spins, there is at least one alignment direction $\boldsymbol{n}_{i}$ of the other spin satisfying the factorization Eqs. (4), given by

$$
\boldsymbol{n}_{i}=\alpha\left[\boldsymbol{a} \times \boldsymbol{b} \pm\left(\eta \lambda_{+} \boldsymbol{a}+\lambda_{-} \boldsymbol{b}\right)\right],
$$

where $\boldsymbol{a}=\mathcal{J}^{i j} \boldsymbol{n}_{j}^{x^{\prime}}, \boldsymbol{b}=\mathcal{J}^{i j} \boldsymbol{n}_{j}^{y^{\prime}}$,

$$
\lambda_{ \pm}^{2}=\frac{\sqrt{\left(|\boldsymbol{a}|^{2}-|\boldsymbol{b}|^{2}\right)^{2}+4|\boldsymbol{a} \cdot \boldsymbol{b}|^{2}} \pm\left(|\boldsymbol{a}|^{2}-|\boldsymbol{b}|^{2}\right)}{2},
$$

and $\alpha$ is a normalization factor, with $\eta=1$ if $\boldsymbol{a} \cdot \boldsymbol{b} \geqslant 0$ and -1 otherwise (if $\boldsymbol{a} \cdot \boldsymbol{b}=0, \lambda_{+}$or $\lambda_{-}$vanishes and the sign of $\eta$ becomes irrelevant). Each sign in Eq. (10) originates a distinct solution for $\boldsymbol{n}_{i}$ (Fig. 2) if $\lambda_{ \pm}$are not both zero. If $\boldsymbol{b} \propto \boldsymbol{a}$ and $\boldsymbol{a} \neq \mathbf{0}$, then $\boldsymbol{n}_{i} \propto \boldsymbol{a}$. Equation (10) holds if $\boldsymbol{a}$ and $\boldsymbol{b}$ are not both zero (see the Appendix for the proof and additional details, including the special case $\boldsymbol{a}=\boldsymbol{b}=\mathbf{0}$ ).

This lemma implies that at least in open one-dimensional systems of $N$ spins with arbitrary, not necessarily uniform first-neighbor quadratic couplings, a fully separable eigenstate, compatible with a given (arbitrary) spin alignment direction of one of the spins, always exists for suitable fields at each site. The alignment directions of the remaining spins are determined by successive applications of this lemma, while the fields are determined by Eqs. (5) and (6). Furthermore, there are typically $2^{N-1}$ configurations of spin directions compatible with the couplings and the initial $\boldsymbol{n}_{j}$, as illustrated in Fig. 2.

Lemma 4. For a pair of equal spins $\left(S_{i}=S_{j}=S\right)$ interacting through $X Y Z$ couplings, if Eqs. (8) are satisfied for non-anti-parallel directions $\boldsymbol{n}_{i}$ and $\boldsymbol{n}_{j}$, there always exist parallel fields $\boldsymbol{h}_{\|}^{i j}$ and $\boldsymbol{h}_{\|}^{j i}$ at $i$ and $j$ such that the factorizing field $\boldsymbol{h}_{s}^{i j}$ for the single pair is uniform:

$$
\boldsymbol{h}_{\|}^{i j}+\boldsymbol{h}_{\perp}^{i j}=\boldsymbol{h}_{\|}^{j i}+\boldsymbol{h}_{\perp}^{j i}=\boldsymbol{h}_{s}^{i j} .
$$

In the uniform case $\boldsymbol{n}_{i}=\boldsymbol{n}_{j}$, it is apparent from Eq. (6) that the perpendicular fields are equal, entailing that $h_{\|}^{i j}=h_{\|}^{j i}$ with the strength $h_{\|}^{i j}$ remaining arbitrary. However, when $\boldsymbol{n}_{i} \neq \boldsymbol{n}_{j}$, Eq. (12) leads to fixed values of the parallel fields $h_{\|}^{i j}, h_{\|}^{j i}$ (explicitly determined in the Appendix) and the pair uniform factorizing fields $\boldsymbol{h}_{s}^{i j}$ belong to the ellipsoid $(\mu \neq \nu \neq \sigma)$ :

$$
\sum_{\mu=x, y, z} \frac{\left(h_{s \mu}^{i j}\right)^{2}}{\left(J_{\mu}^{i j}+J_{v}^{i j}\right)\left(J_{\mu}^{i j}+J_{\sigma}^{i j}\right)}=S^{2} .
$$
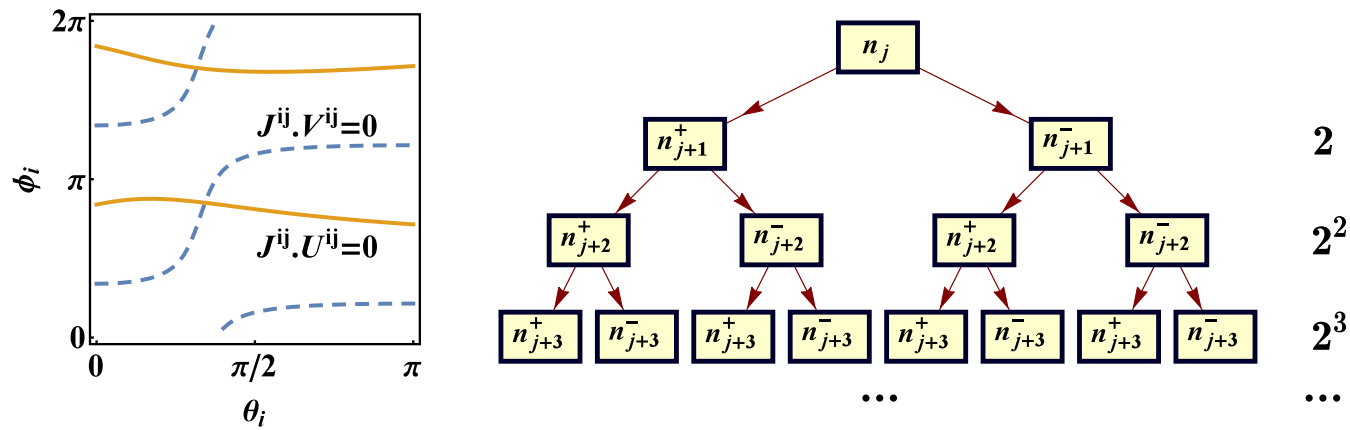

FIG. 2. Left: Typical contour plot of Eqs. (8) for fixed $\boldsymbol{J}^{i j}$ and $\boldsymbol{n}_{j}\left(\boldsymbol{J}^{i j}=J(1,0.75,-0.2), \theta_{j}=\pi / 3, \phi_{j}=\pi / 5\right)$. The two intersection points correspond to the two solutions $\boldsymbol{n}_{i}^{ \pm}$for $\boldsymbol{n}_{i}$ given by Eq. (10). Right: Schematic representation of the $2^{N-1}$ configurations of $|\Theta\rangle$ compatible with the exchange vectors $\boldsymbol{J}^{i j}$ for a spin chain of $N$ spins and an initial alignment direction at site $j$. 
This equation is just that determined by Kurmann et al. in [25] for the Néel-type separable GS in an antiferromagnetic cyclic chain with first-neighbor couplings in a uniform field. Hence, for a uniform field we recover this result. Note, however, that under a uniform field such state will be twofold degenerate if $\boldsymbol{n}_{i} \neq \boldsymbol{n}_{j}$, due to breaking of permutational symmetry [36], and addition of local nonuniform parallel fields is necessary to split this degeneracy.

Lemma 5. Pairwise entanglement reaches full range in the vicinity of factorization.

This result, proved in the Appendix, extends a previous result shown for uniform couplings and fields [36] to the present general case of nonuniform fields and couplings. It means that pairwise entanglement, though obviously vanishing at a separable eigenstate, reaches full range if either the fields or the couplings are slightly varied around the factorization values. It holds for any number $N$ of spins and any spin $S>0$. Factorization can then be also considered as an entanglement critical point in the present general setting.

\section{EXAMPLES}

As illustration of the previous lemmas, we discuss here some special examples of separable eigenstates and show explicit results for the pairwise entanglement in the vicinity of the present general factorization conditions.

\section{A. Spin spiral and other separable eigenstates}

We consider from the present perspective (i.e., starting from the state and deriving the compatible couplings and fields) three examples of separable eigenstates: (i) constant $\theta\left(\theta_{i}=\theta\right.$ in all alignment directions $\boldsymbol{n}_{i}$ ), which includes in particular spin spiral-type eigenstates; (ii) constant $\phi\left(\phi_{i}=\phi \forall \boldsymbol{n}_{i}\right)$; and (iii) uniform (constant $\theta$ and $\phi$ ).

(i) Let us first consider $\theta_{i}=\theta$ for all spins, with $\phi_{i}$ arbitrary. If $\boldsymbol{U}^{i j}, \boldsymbol{V}^{i j}$ are linearly independent, which implies here that $\boldsymbol{n}_{i}+\boldsymbol{n}_{j}$ does not belong to a principal plane, Eqs. (8) or (A1) lead to an $X X Z$ coupling,

$$
J_{x}^{i j}=J_{y}^{i j}=J^{i j}, \quad J_{z}^{i j}=J^{i j} \cos \left(\phi_{i}-\phi_{j}\right),
$$

with $J^{i j}$ arbitrary, which is independent of both $\theta$ and the average $\left(\phi_{i}+\phi_{j}\right) / 2$. From Eq. (6) it can be seen that the perpendicular factorizing fields belong to the principal plane $x y: \boldsymbol{h}_{\perp}^{i j}=J^{i j} S_{j} \sin \left(\phi_{i}-\phi_{j}\right)\left(\boldsymbol{e}_{z} \times \boldsymbol{n}_{i}\right)$.

In particular, considering now a one-dimensional chain with first-neighbor couplings, this case admits solutions with uniform anisotropy $J_{z}^{i j} / J^{i j}=\cos \Delta \phi$, and hence also uniform couplings if $\Delta \phi=\phi_{i+1}-\phi_{i}$ is constant. In a cyclic chain we should have in addition $\Delta \phi=2 \pi k / N$, with $k$ any integer between 1 and $N-1$, as schematically shown in Fig. 3 . For a spin $S$ chain the total perpendicular factorizing fields $\boldsymbol{h}_{\perp}^{i}=\boldsymbol{h}_{\perp}^{i, i-1}+\boldsymbol{h}_{\perp}^{i, i+1}$ become

$$
\boldsymbol{h}_{\perp}^{i}=\left(J^{i-1, i}-J^{i, i+1}\right) S \sin \Delta \phi\left(\boldsymbol{e}_{z} \times \boldsymbol{n}_{i}\right) .
$$

Equation (15) shows that for uniform couplings $\left(J^{i, i+1}=J\right.$ $\forall i), \boldsymbol{h}_{\perp}^{i}=\mathbf{0}$ and the spin spiral is an exact eigenstate of the present $X X Z$ cyclic chain already at zero field (in the open case, the end-point fields $\boldsymbol{h}_{\perp}^{1}$ and $\boldsymbol{h}_{\perp}^{N}$ remain nonzero). In the cyclic case it corresponds to a highly degenerate eigenvalue of $H$ that arises when $J_{z}^{i j} / J^{i j}=\cos \frac{2 \pi k}{N}$. In the presence of parallel fields $\boldsymbol{h}_{\|}^{i}=h_{\|} \boldsymbol{n}_{i}$, the degeneracy will be removed, its energy becoming

$$
E_{\Theta}=-N S\left(h_{\|}+J S \cos \Delta \phi\right) .
$$

It will then be a nondegenerate GS if $h_{\|}$is sufficiently large, typically $h_{\|}=O(|J| S)$.

(ii) Let us consider now $\phi_{i}=\phi$ for all spins, with the angles $\theta_{i}$ remaining arbitrary. Assuming again $\boldsymbol{U}^{i j}$ and $\boldsymbol{V}^{i j}$ linearly independent, i.e., that $\boldsymbol{n}_{i}+\boldsymbol{n}_{j}$ does not belong to a principal plane, Eq. (A1) leads again to an $X X Z$-type coupling,

$$
J_{x}^{i j}=J_{y}^{i j}=J^{i j}\left(1-\eta_{i j}^{2}\right), \quad J_{z}^{i j}=J^{i j}\left(1+\eta_{i j}^{2}\right),
$$

where $\eta_{i j}=\sin \left(\theta_{j}-\theta_{i}\right) / \sin \bar{\theta}_{i j}$, with $\bar{\theta}_{i j}=\left(\theta_{i}+\theta_{j}\right) / 2$ and $J^{i j}$ arbitrary. Hence, the coupling is independent of $\phi$ but depends now on $\theta_{i}, \theta_{j}$, with $\left|J_{z}^{i j}\right| \geqslant\left|J^{i j}\right|$. For small $\theta_{i}-\theta_{j}=\delta \theta$, the orthogonal fields are $\boldsymbol{h}_{\perp}^{i j} \approx$ $-J^{i j} S_{j} \delta \theta \cos \bar{\theta}_{i j}\left(\cos \phi, \sin \phi,-\tan \bar{\theta}_{i j}\right)$, which belongs to the plane defined by $\boldsymbol{n}_{i}$ and $\boldsymbol{n}_{j}$. In a one-dimensional chain with first-neighbor couplings, a constant coupling becomes feasible for a Néel-type configuration with alternating angles $\theta_{1} \theta_{2} \theta_{1} \ldots$, since in this case $\bar{\theta}_{i j}$ and $\left|\theta_{i}-\theta_{j}\right|$ are constant. The energy $E_{\Theta}$ is in any case independent of $\phi$, with $\left\langle\boldsymbol{S}_{i}\right\rangle \cdot \mathcal{J}^{i j}\left\langle\boldsymbol{S}_{j}\right\rangle=S_{i} S_{j} J^{i j}\left[\eta_{i j}^{2} \cos 2 \bar{\theta}_{i j}+\cos \left(\theta_{i}-\theta_{j}\right)\right]$.

(iii) Let us finally consider a fixed alignment direction $\boldsymbol{n}_{i}=\boldsymbol{n}$ for all spins $\left(\theta_{i}=\theta, \phi_{i}=\phi \forall i\right)$. If $\boldsymbol{n}$ does not belong to a principal plane, $\boldsymbol{U}^{i j}$ and $\boldsymbol{V}^{i j}$ are linearly independent and Eqs. (14) or (17) lead to $J_{\mu}^{i j}=J^{i j}$, i.e., to an isotropic coupling $\propto \boldsymbol{S}_{i} \cdot \boldsymbol{S}_{j}$. Equation (A2) then implies $\boldsymbol{h}_{\perp}^{i j}=\mathbf{0}$, i.e., no orthogonal field is required since such uniform state is already an obvious eigenstate of $\boldsymbol{S}_{i} \cdot \boldsymbol{S}_{j}$ for any orientation $\boldsymbol{n}$.

If $\boldsymbol{n}$ belongs instead to a principal plane $\mu \nu\left(n_{\sigma}=0\right), \boldsymbol{V}^{i j}=$ $\mathbf{0}$ and anisotropic couplings become also feasible, provided $\boldsymbol{J}$

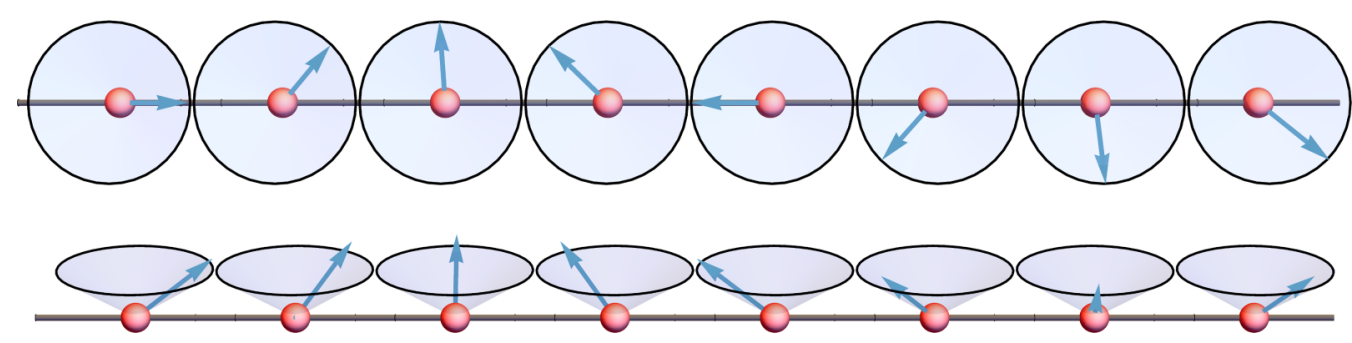

FIG. 3. Schematic representation of the spin spiral separable eigenstate in a spin chain. The alignment direction of the spin at site $i$ is determined by $\theta_{i}=\theta$ and $\phi_{i}=\phi_{1}+(i-1) \Delta \phi \forall i$. The top (bottom) panel corresponds to a top (side) view. 

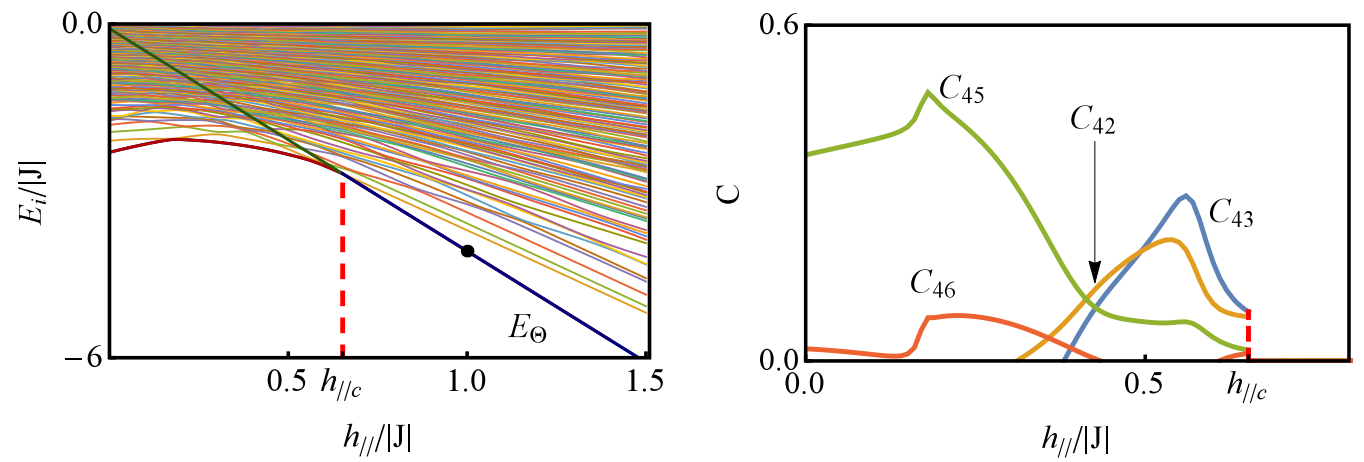

FIG. 4. Left panel: Scaled energy spectrum of a finite spin- $1 / 2$ chain with first-neighbor $X Y Z$ couplings and eight spins when a factorizing field $\boldsymbol{h}^{i}=\boldsymbol{h}_{\perp}^{i}+h_{\|} \boldsymbol{n}^{i}$ with $\boldsymbol{h}_{\perp}^{i}$ fixed and $h_{\|} \geqslant 0$ is applied. The decreasing straight line represents the energy $E_{\Theta}$ of the separable eigenstate $|\Theta\rangle$, which becomes GS for $h_{\|}>h_{\| c}$ (dashed line). Right panel: GS concurrences $C_{i j}$ between a central spin and first and second neighbors, showing that for $h_{\|}<h_{\| c}$ the GS is entangled whereas for $h_{\|}>h_{\| c}$ it is completely separable. All labels are dimensionless.

is orthogonal to $\boldsymbol{U}^{i j}$. This condition leads to

$$
J_{\sigma}^{i j}=J_{\mu}^{i j} n_{v}^{2}+J_{v}^{i j} n_{\mu}^{2}=J_{v}^{i j}+\left(J_{\mu}^{i j}-J_{v}^{i j}\right) \cos ^{2} \gamma
$$

with $J_{\mu}^{i j}, J_{v}^{i j}$ arbitrary and $\gamma$ the angle between $\boldsymbol{n}$ and the $\mu$ axis, implying a fixed ratio $\frac{J_{\sigma}^{i j}-J_{v}^{i j}}{J_{\mu}^{i j}-J_{v}^{i j}}[36]$. The factorizing fields belong to the same principal plane, with

$$
\boldsymbol{h}_{\perp}^{i}=\sin \gamma \cos \gamma\left(\boldsymbol{e}_{\sigma} \times \boldsymbol{n}\right) \sum_{j} S_{j}\left(J_{\mu}^{i j}-J_{v}^{i j}\right)
$$
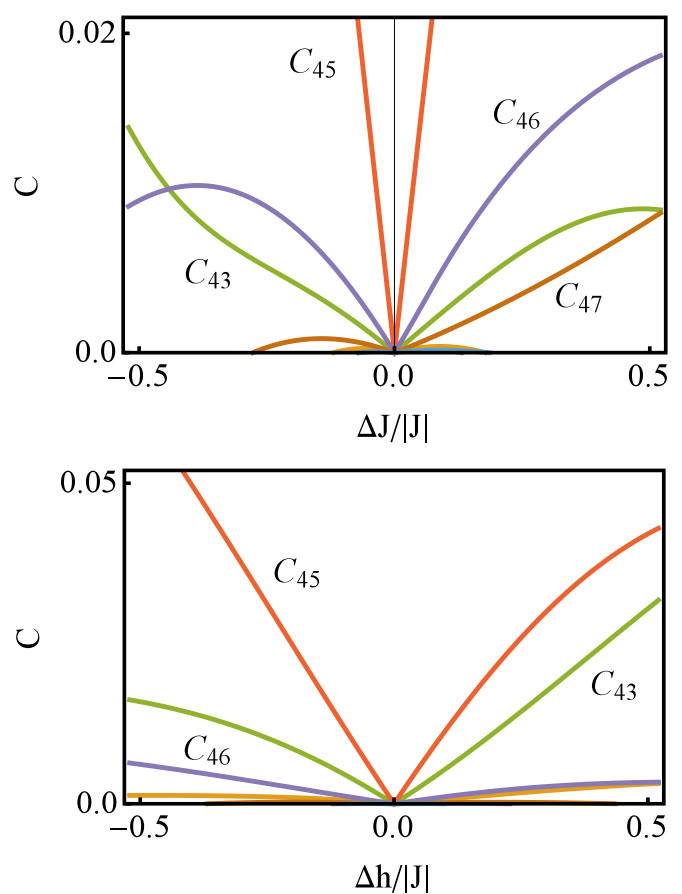

\section{B. Pairwise entanglement}

We now show in Figs. 4 and 5 the behavior of pairwise entanglement in the GS of a finite spin- $1 / 2$ chain with nonuniform first-neighbor couplings under nonuniform fields. The entanglement between spins $i$ and $j$ is measured through the concurrence [48] $C_{i j}=2 \lambda_{\max }-\operatorname{Tr} M_{i j}$ where $\lambda_{\max }$ is the largest eigenvalue of $M_{i j}=\left[\rho_{i j}^{1 / 2} \tilde{\rho}_{i j} \rho_{i j}^{1 / 2}\right]^{1 / 2}$, with $\tilde{\rho}_{i j}=$ $\sigma_{y} \otimes \sigma_{y} \rho_{i j}^{*} \sigma_{y} \otimes \sigma_{y}$ in the standard basis and $\rho_{i j}$ the reduced state of spins $i$ and $j$.

We consider a completely separable eigenstate with the spin alignment directions of the spins selected at random. The exchange couplings between every adjacent pair were then obtained through Eq. (9), setting a uniform norm
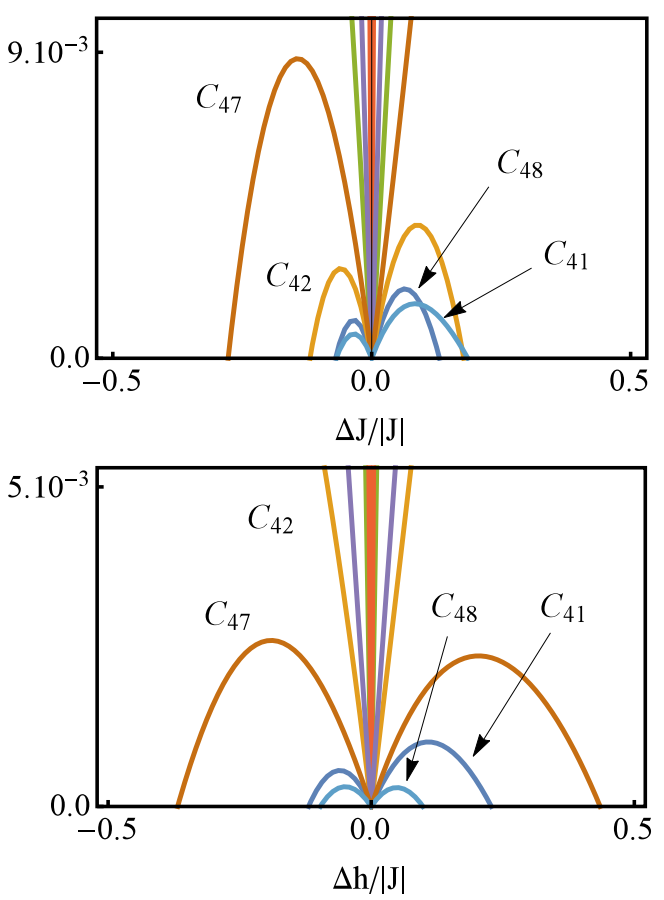

FIG. 5. GS concurrences $C_{i j}$ between spins $i$ and $j$ in the chain of Fig. 4 in the vicinity of factorization (point at $h_{\|} /|\boldsymbol{J}|=1$ on Fig. 6). In the top panel, we have set the couplings as $J_{\mu}^{i j}+\Delta J \forall i, j$, with $J_{\mu}^{i j}$ the factorizing values, such that $C_{i j}=0 \forall i, j$ if $\Delta J=0$. In the bottom panel, the couplings are fixed at the factorizing values but the fields are now $\boldsymbol{h}^{i}+\Delta h \boldsymbol{n}_{i \perp}$, with $\boldsymbol{n}_{i \perp} \propto \boldsymbol{h}_{\perp}^{i}$, such that $C_{i j}=0 \forall i, j$ at $\Delta h=0$. $C_{i j}$ is verified to reach full range in the vicinity of the present general factorization point (nonuniform couplings and fields). Right panels show the same quantities of the left panels at a smaller scale. All labels are dimensionless. 
$\left|J^{i j}\right|=J$ for all exchange vectors. In order for $|\Theta\rangle$ to be a GS, nonuniform fields $\boldsymbol{h}^{i}=\boldsymbol{h}_{\perp}^{i}+h_{\|} \boldsymbol{n}^{i}$, with $\boldsymbol{h}_{\perp}^{i}$ the fixed orthogonal factorizing fields (6) and $h_{\|} \geqslant 0$, were applied at each site. At $h_{\|}=0|\Theta\rangle$ is an exact eigenstate of $H$ although not the GS. As shown in the left panel of Fig. 4, the energy $E_{\Theta}$, given by Eq. (7) decreases linearly (and with maximum slope) for increasing $h_{\|}$, and at $h_{\|}=h_{\| c}$ a GS transition occurs, such that $|\Theta\rangle$ becomes GS $\forall h_{\|}>h_{\| c}$. Accordingly, GS pairwise concurrences $C_{i j}$ vanish for $h_{\|}>h_{\| c} \forall i, j$, as seen in the right panel of Fig. 4.

The behavior of pairwise concurrence in the vicinity of factorization is shown in Fig. 5. We have chosen a stable factorized GS (the point at $h_{\|} /|\boldsymbol{J}|=1$ in Fig. 4), such that the perturbations considered led to a smooth variation of the GS, without crossings with the first excited state. The correction to the field was chosen perpendicular to the alignment direction as any perturbation $\Delta h_{\|} \boldsymbol{n}_{i}$ just shifts the GS energy. It is verified that all concurrences $C_{i j}$ are turned on in the immediate vicinity of factorization for variations of the couplings (top panel) or fields (bottom panel), although those for distant pairs can be very small and vanish outside a small interval. Nonetheless, the factorization point stands out as an entanglement "critical point" of the system, in the sense of exhibiting infinite range in its vicinity. Note also that coefficients $\beta_{i j}$ in the reduced state of the pair, Eq. (A11), will vanish and hence change their signs at $\Delta J=0$ or $\Delta h=0$.

\section{SEPARABLE GROUND-STATE ENGINEERING}

One of the goals of this paper is to provide recipes for engineering nondegenerate maximally aligned exactly separable GS in spin systems. In the previous section this problem was approached from two different perspectives: (I) specifying the alignment directions $\boldsymbol{n}_{i}$ of the spins and finding compatible exchange vectors $\boldsymbol{J}^{i j}$ (Lemma 2) and (II) assuming fixed exchange couplings $\boldsymbol{J}^{i j}$ and finding compatible alignment directions of the spins (Lemma 3 ). The first scheme has, for instance, enabled us to easily identify spin-spiral-type separable eigenstates in $X X Z$ chains with special values of $J_{z} / J_{x}$, already at zero field.

In the first case it is evident that a necessary condition for engineering the separable GS is that the exchange coupling between the spins must be tunable. This could in principle be feasible in spin systems based on quantum dots [41,42], superconducting Josephson junctions [43], and nuclear (or electron-nuclear) spin states [44]. In the second scenario the exchange couplings are fixed and Lemma 3 yields the possible separable eigenstates the system can possess. This is a more restrictive case, as we suppose little (to none) control over the exchange couplings. Thus, according to how much control is available over the system the problem may be considered from one standpoint or the other. To quantify such control (and assuming that a uniform field can always be applied) we introduce the experimental complexity " $\varepsilon_{c}=(\boldsymbol{m}, \boldsymbol{k})$," which indicates that for a system of $N$ interacting spins to have a given separable state as its nondegenerate GS, control over $m \leqslant N-1$ local fields and $k$ exchange couplings between spins is required. As expected, the separable state which requires the simplest control will be shown to be the uniform separable state.

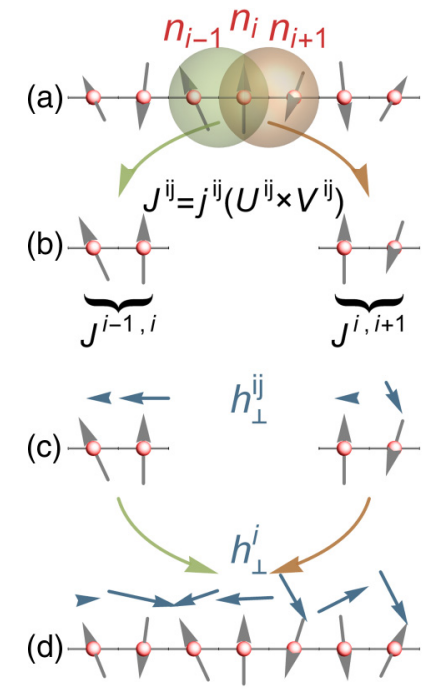

(a)

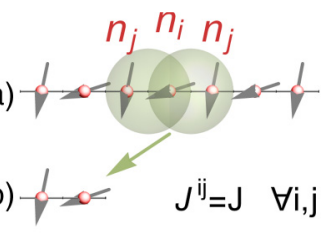

(c)

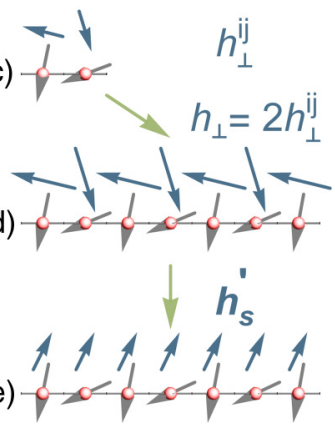

FIG. 6. Schematic representation of the separable state with arbitrary spin alignment directions $\boldsymbol{n}_{i}$ at each site (left panel) and with a Néel-type configuration (right panel). (a) The alignment directions at each site are specified. Then, for each pair the (b) exchange couplings and (c) perpendicular fields are determined. (d) The perpendicular factorizing field at each site is $\boldsymbol{h}_{\perp}^{i}=\sum_{j} \boldsymbol{h}_{\perp}^{i j}$. (e) The uniform factorizing field $\boldsymbol{h}_{s}^{\prime}$ is determined by Lemma 4 .

\section{A. Tunable exchange couplings}

As shown in Lemma 2, by specifying the individual spin alignments $\boldsymbol{n}_{i}$ and $\boldsymbol{n}_{j}$ of an interacting pair, the exchange vector $\boldsymbol{J}^{i j}$ and the fields $\boldsymbol{h}_{\perp}^{i j}, \boldsymbol{h}_{\perp}^{j i}$ can be determined. Then, by applying suitable parallel fields at each site, the separable state $|\Theta\rangle$ can be made a nondegenerate GS of $H$ (Lemma 1). Assuming that a uniform field can be applied, then $\boldsymbol{\varepsilon}_{\boldsymbol{c}}=(\mathbf{1 , 1})$ for a single pair. Similarly, for a chain of $N$ spins with first-neighbor couplings, $\varepsilon_{c}=(N-1, N-1)$ in the open case and $\varepsilon_{c}=(N-1, N)$ in the cyclic case (Fig. 6).

A possible way of reducing the complexity is to obtain separability by only applying a uniform factorizing field. According to Lemma 4, this is always possible in a system of two identical spins if Eqs. (8) are fulfilled, provided the alignment directions $\boldsymbol{n}_{1}$ and $\boldsymbol{n}_{2}$ are not antiparallel. Nonetheless, if $\boldsymbol{n}_{1} \neq \boldsymbol{n}_{2}$ such eigenstate will be twofold degenerate (due to basic permutational symmetry breaking) and local nonuniform parallel fields must be added to split this degeneracy. Therefore, the complexity remains unchanged if a nondegenerate GS is to be achieved. The same holds for finite cyclic chains with first-neighbor couplings and an even number of spins if an alternating Néel-type separable eigenstate $\left[\boldsymbol{n}_{i}=\boldsymbol{n}_{1}\left(\boldsymbol{n}_{2}\right)\right.$ for $i$ odd (even)] is sought. With the same previous scheme (and just doubling the field at each site due to the contributions from each neighbor) it is possible to obtain such eigenstate by applying a uniform field (right panel in Fig. 6). This state can be a GS for antiferromagnetic-type couplings [25], although it will be again degenerate. An additional alternating field will then be required to turn it into a nondegenerate GS.

On the other hand, if $S_{i}=S_{j}$ and $\boldsymbol{n}_{i}=\boldsymbol{n}_{j}$, the uniform factorizing field is just $\boldsymbol{h}_{s}=\boldsymbol{h}_{\|}+\boldsymbol{h}_{\perp}$ with the strength $h_{\|}$ remaining arbitrary. Thus, according to Lemma 1 it is possible 
to make this state a nondegenerate GS with an arbitrarily large spectral gap by applying just a uniform field $\boldsymbol{h}_{s}$, implying $\boldsymbol{\varepsilon}_{c}=(\mathbf{0}, \mathbf{1})$. Similarly, in a cyclic chain of $N$ (even or odd) spins with first-neighbor couplings, such states require $\varepsilon_{c}=(\mathbf{0}, N)$, due to the same arguments. In an open chain it is necessary, however, to correct the fields at the borders due to one missing neighbor and hence $\boldsymbol{\varepsilon}_{c}=(\mathbf{2}, \boldsymbol{N}-\mathbf{1})$.

Achieving the necessary control over the exchange interactions and local magnetic fields is a challenge in itself. However, this requirement can be relaxed by considering spin clusters schemes [45] where the qubit is encoded in several spins. The previous schemes can then be used as building blocks to engineer (bulk per bulk) the separable GS. If the spin configuration of each cluster is uniform, the factorizing fields at each bulk will also be uniform, and we would only require control over the exchange couplings and fields at the border of the clusters.

\section{B. Fixed exchange couplings}

For a pair of interacting spins, given the alignment direction $\boldsymbol{n}_{j}$ of one of the spins, according to Lemma 3 an alignment direction $\boldsymbol{n}_{i}$ of the remaining spin can always be determined, regardless of the coupling between them. Then, by appropriate fields the ensuing separable state can be made an exact GS with $\boldsymbol{\varepsilon}_{\boldsymbol{c}}=(\mathbf{1 , 0 )}$. In finite arrays Lemma 3 can therefore be used to determine spin configurations compatible with the fixed exchange couplings. For instance, in an open chain of $N$ spins with first-neighbor couplings, by specifying the alignment direction of only one spin, this method determines the possible alignment directions of the remaining spins (typically $2^{N-1}$ configurations, Fig. 2, right panel). This scheme is represented in the left panel of Fig. 7. In this case $\varepsilon_{c}=(N-\mathbf{1 , 0})$, whereas (a)

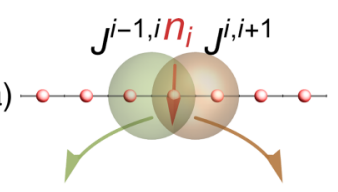

(b)<smiles>CC(C)(C)C(C)(C)C(C)(C)C</smiles>

$n_{i-1}$

(c)

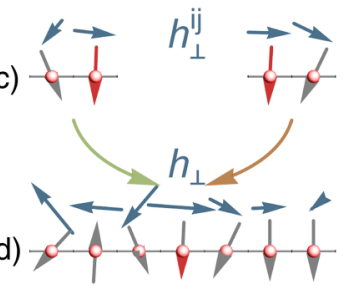

(a)

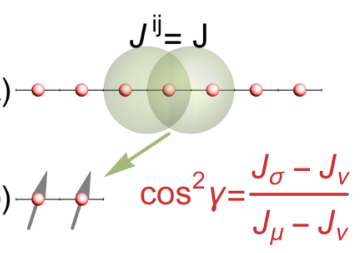

(c)<smiles>CC1(C)CCCC1(C)C</smiles>

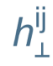

$h_{\perp}=2 h_{\perp}^{\mathrm{ij}}$

(d)

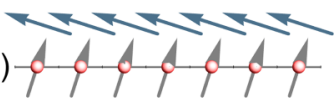

FIG. 7. Schematic representation of a spin chain with nonuniform couplings (left panel) and with uniform anisotropic couplings leading to a uniform separable state (right panel). (a) The exchange couplings are fixed. In the left panel one of the spin alignment directions $\boldsymbol{n}_{i}$ is specified. (b) Left: Using Lemma 3 the spin alignment directions for the spins at sites $i \pm 1$ can be determined. Right: $\boldsymbol{n}$ belongs to the principal plane $\mu \nu$ determined by the exchange couplings. (c,d) Left: By successive applications of Lemma 3 the spin alignments of the remaining spins are determined. in the cyclic case control on one exchange coupling is required, meaning that $\boldsymbol{\varepsilon}_{c}=(N-\mathbf{1 , 1})$.

If in the previous system the exchange couplings are uniform, $\boldsymbol{J}^{i j}=\boldsymbol{J}$, the uniform separable solution $\boldsymbol{n}_{i}=\boldsymbol{n} \forall i$ is always feasible provided $\boldsymbol{n}$ is appropriately chosen. If the coupling is isotropic, $J_{\mu}=J, \mu=x, y, z$, then, as discussed in the previous section, $\boldsymbol{n}$ is arbitrary, i.e., the solution for $\boldsymbol{n}_{i}$ given by Lemma 3 is the same $\boldsymbol{n}_{j}$ of the initial spin for any $\boldsymbol{n}_{j}$ (see the Appendix). However, if the exchange interaction is anisotropic, a uniform solution is feasible provided $\boldsymbol{n}_{j}$ belongs to a principal plane and satisfies Eq. (18), as depicted in the right panel of Fig. 7. In cyclic chains (with either isotropic or anisotropic couplings) such uniform $|\Theta\rangle$ can then be made a nondegenerate GS with just a uniform magnetic field, i.e., $\boldsymbol{\varepsilon}_{\boldsymbol{c}}=(\mathbf{0 , 0})$, while in open chains $\boldsymbol{\varepsilon}_{\boldsymbol{c}}=\mathbf{( 2 , 0 )}$ due to the border corrections. On the other hand, Néel-type solutions, also feasible for uniform first-neighbor couplings, require an additional alternating field in order to become a nondegenerate GS. The uniform solution is also directly feasible for higher range couplings [36], as well as in more general arrays and geometries. Just the fields near the border should be adequately corrected. The uniform separable GS is therefore that requiring the least control over the system.

\section{Experimental feasibility and implementations}

The possibility of simulating interacting spin systems enables the aforementioned engineering methods to be realized. We mention two physical realizations, in which, with the current state of technology, couplings and fields can be controlled.

\section{Superconducting devices}

Superconducting qubits based on Josephson junctions in solid-state electrical circuits present an attractive scenario for a quantum simulator due to their robustness, long coherence times, and intrinsic low dissipation [15]. It has been shown that superconducting flux qubits circuits can be used (under specific regimes) to simulate $X X$ spin systems with nearest-neighbor interactions with nontransverse nonuniform fields (belonging to the $x z$ principal plane of the couplings) [16]. In these systems, the exchange couplings can be tuned if a direct current superconducting quantum interference device (SQUID) is used to couple the flux qubits [17], whilst the direction and strength of the local magnetic fields are controlled by the phases and amplitudes, respectively, of microwave driving fields. Realizations of chains with first-neighbor tunable $X Z$ couplings, not necessarily uniform, in nontransverse and nonuniform fields have also been recently reported using planar transmon qubits (a type of superconducting charge qubits) [18].

\section{Trapped ions}

When interacting with lasers, trap ions can simulate $X Y Z$ effective spin systems in nontransverse magnetic fields. Trapped ions methods are highly controllable and versatile and present long decoherence times and high readout precision. When simulating interacting spin systems, the exchange 
couplings and the effective magnetic fields can be tuned by controlling the lasers acting on the internal transition of the ions [19,24].

In these systems, once the nondegenerate separable GS is obtained it can be used in actual computing instances which require an initial fully separable state, as in standard models for quantum computation and quantum simulation [1,14,24,37]. In particular, in typical quantum annealing, one starts from a known separable GS of a trivial noninteracting Hamiltonian (usually $h \sum_{i} S_{i}^{x}$ ) which is then continuously driven to a target interacting Hamiltonian whose nontrivial GS is sought [39,40] (normally an Ising type Hamiltonian $h^{\prime} \sum_{i} S_{i}^{z}+\sum_{i, j} J^{i j} S_{i}^{z} S_{j}^{z}$ ). Thus, the previous factorization schemes enable us to think of annealing protocols with always-on interactions in which just a part of the original Hamiltonian is quenched. For instance, one could start from a convenient maximally aligned uniform separable GS in an $X Y Z$ system with a nontransverse field, such that the GS is well gapped, and continuously decrease the field along the $x$ axis and the $X Y$ terms leaving the sought Ising Hamiltonian. Additionally, quantum annealing could be used in principle to obtain the GS of a complex Hamiltonian starting from a separable GS by an analogous process (i.e., just modifying the fields, initially at suitable factorizing values). In particular, in chains with fixed arbitrary first-neighbor couplings, Lemmas 3 and 1 ensure the existence of (multiple) completely separable gapped GSs if the fields are adequately tuned, entailing that the previous annealing could always be applied.

\section{CONCLUSIONS}

We have presented an approach to the problem of factorization, showing the possibility of starting from the state and determining the compatible couplings and fields. This approach opens the way to separable ground-state engineering in interacting spin systems if some control over the couplings (assumed quadratic) and fields is feasible. For a fully separable state with arbitrary spin alignment directions at each site, nonzero couplings between any selected pairs (which can be all pairs or just some pairs) and finite fields at each site always exist such that the ensuing Hamiltonian has such state as a nondegenerate GS. In this way, some noticeable separable eigenstates (like the spin-spiral solution) were easily identified in $X X Z$ chains. In addition, in open one-dimensional systems of $N$ spins with arbitrary first-neighbor couplings, at least one (and typically many) exactly separable GS compatible with an arbitrary spin direction at one site is always feasible if the fields can be tuned at each site. And for a single pair, the field can always be chosen as uniform. Furthermore, pairwise entanglement reaches full range in the immediate vicinity of factorization (for perturbations in the fields or couplings), regardless of the type of solution, indicating that even in the present general setting factorization can still be considered as an entanglement critical point. These results, while providing useful insight into interacting spin systems and models without analytical solution, enable us to devise separable groundstate engineering methods which could be used in quantum information protocols and quantum annealing.

\section{ACKNOWLEDGMENTS}

The authors acknowledge support from CONICET (M.C., N.C.) and CIC (R.R.) of Argentina. Work was supported by CONICET PIP Grant No. 112201501-00732.

\section{APPENDIX}

We present here proof details of the lemmas presented in Sec. II B.

Lemma 1. As previously stated, there is no state $|\Psi\rangle$ orthogonal to $|\Theta\rangle$ whose energy decreases more rapidly with $h_{\|}^{i}$ than $E_{\Theta}$. For instance, if $S_{i}=S \forall i,\left|\left\langle S_{i} \cdot \mathcal{J}^{i j} S_{j}\right\rangle_{\Psi}\right| \leqslant J^{i j} S(S+1)$ is bounded (with $J^{i j}$ the maximum singular value of the matrix $\mathcal{J}^{i j}$ ) while $\left\langle-\sum_{i} \boldsymbol{S}_{i} \cdot \boldsymbol{h}^{i}\right\rangle_{\Psi} \geqslant-(N-1) S h_{\|}-\sum_{i}\left\langle\boldsymbol{S}_{i}\right\rangle_{\Psi} \cdot \boldsymbol{h}_{\perp}^{i}$ if $\boldsymbol{h}^{i}=h_{\|} \boldsymbol{n}_{i}+\boldsymbol{h}_{\perp}^{i}$ and $N$ is the number of spins. Hence, no state $|\Psi\rangle$ can match the decrease with increasing $h_{\|}$of $E_{\Theta}$, which will contain a term $\propto-N S h_{\|}$. Typically, if all $J^{i j}$ are $O(J)$, the threshold parallel field $h_{\| c}$ for making $|\Theta\rangle$ a GS will be $O(J S l)$, with $l$ the number of neighbors coupled with a given spin.

Lemma 2. If $\boldsymbol{J}^{i j}$ is orthogonal to the subspace spanned by the vectors $\boldsymbol{U}^{i j}$ and $\boldsymbol{V}^{i j}$, Eqs. (8) are satisfied. Here we explicitly determine these exchange couplings and the concomitant factorizing fields for the two different situations that need to be considered.

(a) $\boldsymbol{U}^{i j}$ and $\boldsymbol{V}^{i j}$ linearly independent. In this case $\boldsymbol{J}^{i j}$ is given by Eq. (9), which can be explicitly written in terms of the alignment directions $\boldsymbol{n}_{i}, \boldsymbol{n}_{j}$ as

$$
J_{\mu}^{i j}=-j^{i j}\left(n_{j \mu} D_{i} / n_{i \mu}+n_{i \mu} D_{j} / n_{j \mu}\right),
$$

where $D_{i}=\prod_{\mu} n_{i \mu}=\frac{1}{4} \sin \theta_{i} \sin 2 \theta_{i} \sin 2 \phi_{i}$. It satisfies $J_{\mu}^{i j}=J_{\mu}^{j i}$. The orthogonal fields $\boldsymbol{h}_{\perp}^{i j}$ can then be specified just in terms of the alignment directions and the constants $j^{i j}$ :

$$
\left(\boldsymbol{h}_{\perp}^{i j}\right)_{\mu}=j^{i j} S_{j}\left(n_{j \mu}^{2}-n_{i \mu}^{2}\right) D_{i} / n_{i \mu} .
$$

Correspondingly, the energy $E_{\Theta}$, Eq. (7), becomes

$$
E_{\Theta}=-\sum_{i} S_{i} h_{\|}^{i}-\frac{1}{2} \sum_{i \neq j} j^{i j} S_{i} S_{j}\left(D_{i}+D_{j}\right) .
$$

(b) $\boldsymbol{U}^{i j}$ and $\boldsymbol{V}^{i j}$ linearly dependent. For the present orthogonal vectors $\boldsymbol{n}_{i}^{\mu^{\prime}}$, this case occurs when $\boldsymbol{V}^{i j}=\mathbf{0}$ (and $\boldsymbol{U}^{i j} \neq \mathbf{0}$ ). Hence, Eqs. (8) define a plane orthogonal to $\boldsymbol{U}^{i j}$ of exchange vectors $\boldsymbol{J}^{i j}$.

(i) If $\boldsymbol{n}_{i}$ and $\boldsymbol{n}_{j}$ belong to the same principal plane, say $\mu \nu$, with $\sigma$ the direction orthogonal to this plane $\left(n_{i \sigma}=n_{j \sigma}=0\right)$, Eqs. (8) lead to

$$
J_{\sigma}^{i j}=J_{\mu}^{i j} n_{i \nu} n_{j v}+J_{\nu}^{i j} n_{i \mu} n_{j \mu},
$$

with $J_{\mu}^{i j}$ and $J_{v}^{i j}$ arbitrary. There are now two independent exchange couplings, which are those of the plane containing the alignment directions (bottom right panel in Fig. 1). From Eq. (6) it is seen that $\boldsymbol{h}_{\perp}^{i j}$ also belongs to the principal plane $\mu \nu$, directly depending on the free couplings $J_{\mu}^{i j}$ and $J_{v}^{i j}$. Moreover, by choosing them such that $\boldsymbol{n}_{i} \times\left(\boldsymbol{J}^{i j} * \boldsymbol{n}_{j}\right)=\mathbf{0}$, then $\boldsymbol{h}_{\perp}^{i j}=\mathbf{0}$, i.e., $|\Theta\rangle$ is an exact eigenstate at zero field.

(ii) If $\boldsymbol{n}_{i}$ is the reflection of $\boldsymbol{n}_{j}$ with respect to the principal plane $\mu \nu$, with all components of $\boldsymbol{n}_{i(j)}$ nonzero (otherwise we 
return to previous case i), Eqs. (8) lead to

$$
J_{\sigma}^{i j}=\frac{J_{\mu}^{i j}\left(1-n_{i \mu}^{2}\right)+J_{v}^{i j}\left(1-n_{i v}^{2}\right)}{1-n_{i \sigma}^{2}},
$$

where $J_{\mu}^{i j}$ and $J_{v}^{i j}$ are arbitrary. Then, from Eq. (6) the orthogonal fields are

$$
\begin{gathered}
\left(\boldsymbol{h}_{\perp}^{i j}\right)_{\sigma}=S_{j}\left(J_{\mu}^{i j}+J_{v}^{i j}\right) n_{i \sigma}, \\
\left(\boldsymbol{h}_{\perp}^{i j}\right)_{\mu(v)}=S_{j}\left(J_{v(\mu)}^{i j}-J_{\sigma}^{i j}\right) n_{i \mu(v)} .
\end{gathered}
$$

(iii) Finally, if $\boldsymbol{n}_{i}=-\boldsymbol{n}_{j}$, we should just replace $J_{\sigma}^{i j}$ by $-J_{\sigma}^{i j}$ in Eqs. (A5) and (A6), such that $\boldsymbol{J}^{i j}$ belongs to the plane $\sum_{\mu=x, y, z} J_{\mu}^{i j}\left(1-n_{\mu}^{2}\right)=0$, with $\left(\boldsymbol{h}_{\perp}^{i j}\right)_{\mu}=S_{j}\left[\operatorname{Tr}(\mathcal{J})-J_{\mu}\right] n_{i \mu}$.

A final remark is that if one approaches any of the cases (b) from the linearly independent case (a), it is verified that Eqs. (A4)-(A6) are in agreement with the corresponding limit of Eqs. (A1) and (A2).

Lemma 3. Proof. Assuming first $\boldsymbol{a}=\mathcal{J}^{i j} \boldsymbol{n}_{j}^{x^{\prime}}$ and $\boldsymbol{b}=$ $\mathcal{J}^{i j} \boldsymbol{n}_{j}^{y^{\prime}}$ linearly independent, we can define orthonormal vectors $\boldsymbol{k}, \boldsymbol{l}$ such that $\boldsymbol{a}=|\boldsymbol{a}| \boldsymbol{k}, \boldsymbol{b}=b_{1} \boldsymbol{k}+b_{2} \boldsymbol{l}$, with $b_{1}=$ $\boldsymbol{a} \cdot \boldsymbol{b} /|\boldsymbol{a}|$. Then normalized vectors $\boldsymbol{n}_{i}^{x^{\prime}} \propto b_{2} \boldsymbol{k}-b_{1} \boldsymbol{l}+\lambda \boldsymbol{m}$ and $\boldsymbol{n}_{i}^{y^{\prime}} \propto \lambda \boldsymbol{l}+b_{1} \boldsymbol{m}$, with $\boldsymbol{m}=\boldsymbol{k} \times \boldsymbol{l}$, satisfy $\boldsymbol{n}_{i}^{x^{\prime}} \cdot \boldsymbol{b}=\boldsymbol{n}_{i}^{y^{\prime}} \cdot \boldsymbol{a}=$ $\boldsymbol{n}_{i}^{x^{\prime}} \cdot \boldsymbol{n}_{i}^{y^{\prime}}=0$. Hence, the factorization Eqs. (4) are fulfilled provided $\boldsymbol{n}_{i}^{x^{\prime}} \cdot \boldsymbol{a}=\boldsymbol{n}_{i}^{y^{\prime}} \cdot \boldsymbol{b}$, which implies $\lambda= \pm \lambda_{+}$, with $\lambda_{+}$ given by Eq. (11). A suitable alignment direction at site $i$ can then be obtained as $\boldsymbol{n}_{i}=\boldsymbol{n}_{i}^{x^{\prime}} \times \boldsymbol{n}_{i}^{y^{\prime}}$, which yields Eq. (10).

Additionally, if $\boldsymbol{b} \propto \boldsymbol{a}$, with $\boldsymbol{a} \neq \mathbf{0}$, Eq. (10) still holds, since in this case it leads to $\boldsymbol{n}_{i} \propto \boldsymbol{a}$, which is indeed an obvious solution for $\boldsymbol{n}_{i}$ of Eqs. (4). And if $\boldsymbol{a}=\boldsymbol{b}=\mathbf{0}$, which occurs iff both $\boldsymbol{J}^{i j}$ (assumed nonzero) and $\boldsymbol{n}_{j}$ point along the same principal axis $(\mu)$, then $\boldsymbol{n}_{i}$ remains arbitrary. The effect of the coupling on the product state can here be balanced by a factorizing field, as it involves just one-spin excitations: $J_{\mu}^{i j} S_{i}^{\mu} S_{j}^{\mu}|\Theta\rangle=S_{j} J_{\mu}^{i j} S_{i}^{\mu}|\Theta\rangle$.

As is evident from Eq. (10), two different solutions for $\boldsymbol{n}_{i}$ exist unless $\lambda_{ \pm}$are simultaneously zero. This case arises, for instance, if $\boldsymbol{J}^{i j} \propto(1,1,1)$ (isotropic coupling) or if all components of $\boldsymbol{J}$ have the same absolute value, e.g., $\boldsymbol{J} \propto$ $(1,1,-1)$, which imply $|\boldsymbol{a}|=|\boldsymbol{b}|$ and $\boldsymbol{a} \cdot \boldsymbol{b}=0$ in Eq. (11). In the isotropic case, $\boldsymbol{a}=\boldsymbol{n}_{j}^{x^{\prime}}, \boldsymbol{b}=\boldsymbol{n}_{j}^{y^{\prime}}$ and Eq. (10) implies then the single solution $\boldsymbol{n}_{i}=\boldsymbol{n}_{j}$ (uniform solution).

Lemma 4. Proof. From Eq. (6), $\boldsymbol{h}_{\perp}^{i j}=-S\left[\mathcal{J}^{i j} \boldsymbol{n}_{j}-\boldsymbol{n}_{i}\left(\boldsymbol{n}_{i}\right.\right.$. $\left.\left.\mathcal{J}^{i j} \boldsymbol{n}_{j}\right)\right]$ and Eq. (12) implies

$$
\begin{aligned}
S \boldsymbol{J}^{i j} *\left(\boldsymbol{n}_{j}-\boldsymbol{n}_{i}\right)= & {\left[h_{\|}^{i j}+S\left(\boldsymbol{n}_{i} \cdot \boldsymbol{J}^{i j} * \boldsymbol{n}_{j}\right)\right] \boldsymbol{n}_{\boldsymbol{i}} } \\
& -\left[h_{\|}^{j i}+S\left(\boldsymbol{n}_{j} \cdot \boldsymbol{J}^{i j} * \boldsymbol{n}_{i}\right)\right] \boldsymbol{n}_{\boldsymbol{j}},
\end{aligned}
$$

which is verified for some $h_{\|}^{i j}$ and $h_{\|}^{j i}$ iff $\boldsymbol{J}^{i j} *\left(\boldsymbol{n}_{j}-\boldsymbol{n}_{i}\right)$ belongs to the subspace generated by $\boldsymbol{n}_{i}$ and $\boldsymbol{n}_{j}$. If $\boldsymbol{n}_{i}=\boldsymbol{n}_{j}$ this condition is trivially satisfied (with $h_{\|}^{i j}=h_{\|}^{j i}$, arbitrary) while if $\boldsymbol{n}_{i}$ and $\boldsymbol{n}_{j}$ are not collinear this condition implies $\left[\boldsymbol{J}^{i j} *\left(\boldsymbol{n}_{i}-\boldsymbol{n}_{j}\right)\right] \cdot\left(\boldsymbol{n}_{i} \times \boldsymbol{n}_{j}\right)=0$, i.e., $\boldsymbol{J}^{i j} \cdot\left[\left(\boldsymbol{n}_{i}-\boldsymbol{n}_{j}\right) *\left(\boldsymbol{n}_{i} \times\right.\right.$ $\left.\left.\boldsymbol{n}_{j}\right)\right]=0$. But this equation is always fulfilled if $\boldsymbol{J}^{i j} \propto \boldsymbol{U}^{i j} \times$ $\boldsymbol{V}^{i j}$ [Eq. (9)], while if $\boldsymbol{U}^{i j}$ and $\boldsymbol{V}^{i j}$ are linearly dependent it is fulfilled by any $\boldsymbol{J}^{i j}$, since in this case $\left(\boldsymbol{n}_{i}-\boldsymbol{n}_{j}\right) *\left(\boldsymbol{n}_{i} \times \boldsymbol{n}_{j}\right)=$ 0. No solution exists, however, if $\boldsymbol{n}_{i}=-\boldsymbol{n}_{j}$.
In the last antiparallel case, it is evident from Eq. (6) that $\boldsymbol{h}_{\perp}^{i j}=-\boldsymbol{h}_{\perp}^{j i}$ and hence there are no parallel fields $\boldsymbol{h}_{\|}^{i j(j i)}$ able to lead to a uniform factorizing field for the pair, unless $\boldsymbol{h}_{\perp}^{i j}=\mathbf{0}$ (for instance, antiparallel alignment directions along the $z$ axis fulfill Eqs. (8) if $J_{x}^{i j}=-J_{y}^{i j}$ and lead to $\boldsymbol{h}_{\perp}^{i j}=\mathbf{0}$ ).

As previously discussed, in the uniform case $\boldsymbol{n}_{i}=\boldsymbol{n}_{j}$ the perpendicular fields are equal and $h_{\|}^{i j}=h_{\|}^{j i}$, with the strength of $h_{\|}^{i j}$ remaining arbitrary. However, when $\boldsymbol{n}_{i} \neq \boldsymbol{n}_{j}$ Eq. (A7) will lead to fixed values of the parallel fields, which we now proceed to explicitly determine.

When $\boldsymbol{U}^{i j}$ and $\boldsymbol{V}^{i j}$ are linearly independent, by solving Eq. (A7) it is found that $h_{\|}^{i j}=-j^{i j} S\left[D_{i}+\boldsymbol{n}_{i}\right.$. $\left.\left(n_{j y} n_{j z}, n_{j x} n_{j z}, n_{j x} n_{j y}\right)\right]$. The uniform factorizing field $\boldsymbol{h}_{s}^{i j}=$ $\boldsymbol{h}_{\|}^{i j}+\boldsymbol{h}_{\perp}^{i j}$ becomes then ( $\mu, \nu, \sigma$ indicate three distinct principal axes)

$$
\left(h_{s}^{i j}\right)_{\mu}=-j^{i j} S \alpha_{\mu \nu} \alpha_{\mu \sigma}, \quad \alpha_{\mu \nu}=n_{i \mu} n_{j \nu}+n_{i \nu} n_{j \mu} .
$$

On the other hand, when $\boldsymbol{V}^{i j}=\mathbf{0}$, Eq. (A7) leads to $h_{\|}^{i j}=$ $-S \frac{\boldsymbol{n}_{i} \cdot \boldsymbol{J}^{i j} * \boldsymbol{n}_{i}+n_{i \mu} J_{\mu}^{i j}+n_{j \mu}\left(J_{\sigma}^{i j}+J_{\nu}^{i j}\right)}{n_{i \mu}+n_{j \mu}}$ if $\boldsymbol{n}_{i}$ and $\boldsymbol{n}_{j}$ belong both to the principal plane $\mu \nu$, and $h_{\|}^{i j}=h_{\|}^{j i}=-S\left(J_{\mu}^{i j}+J_{\nu}^{i j}\right)$ if $\boldsymbol{n}_{i}$ is the reflection of $\boldsymbol{n}_{j}$ with respect to the principal plane $\mu \nu$. In this case, Eqs. (A6) lead to

$$
\left(h_{s}^{i j}\right)_{\mu(v)}=-S\left(J_{\mu(\nu)}^{i j}+J_{\sigma}^{i j}\right) n_{i \mu(v)}, \quad\left(h_{s}^{i j}\right)_{\sigma}=0,
$$

meaning that the $\sigma$ components of $\boldsymbol{h}_{\|}^{i j(j i)}$ and $\boldsymbol{h}_{\perp}^{i j(j i)}$ cancel each other such that the local uniform factorizing field belongs to the principal plane $\mu \nu$.

Lemma 5. Proof. Let $\boldsymbol{h}^{i}$ and $\mathcal{J}^{i j}$ be the fields and couplings for which the separable state $|\Theta\rangle$ (not necessarily uniform) is a nondegenerate GS. Then, if $\boldsymbol{h}^{i} \rightarrow \boldsymbol{h}^{i}+\delta \boldsymbol{h}^{i}$ and $J_{\mu \nu}^{i j} \rightarrow$ $J_{\mu \nu}^{i j}+\delta J_{\mu \nu}^{i j}$, the perturbed GS is $|\mathrm{GS}\rangle=|\Theta\rangle+\delta|\mathrm{GS}\rangle$, with

$$
\begin{aligned}
\delta|\mathrm{GS}\rangle & \approx \sum_{\nu} \frac{\left\langle\nu\left|\left(\sum_{i} \delta \boldsymbol{h}_{\perp}^{i} \cdot \boldsymbol{S}_{i}+\sum_{i<j, \mu, \nu} \delta J_{\mu \nu}^{i j} S_{i}^{\mu} S_{j}^{\nu}\right)\right| \Theta\right\rangle}{E_{\nu}-E_{\Theta}}|v\rangle \\
& =\left(\sum_{i} \alpha_{i} S_{i}^{-^{\prime}}+\sum_{i, j} \beta_{i j} S_{i}^{-^{\prime}} S_{j}^{-^{\prime}}+\ldots\right)|\Theta\rangle, \quad(\mathrm{A} 10)
\end{aligned}
$$

up to lowest nonzero order, where $|\nu\rangle$ are the exact excited eigenstates at the factorizing point $\left(H|v\rangle=E_{v}|\nu\rangle,\langle\nu \mid \Theta\rangle=\right.$ $0)$, normally entangled; $\delta \boldsymbol{h}_{\perp}^{i}$ is the component of $\delta \boldsymbol{h}^{i}$ orthogonal to $\boldsymbol{n}_{i}$; and $S_{i}^{ \pm^{\prime}}=R_{i} S_{i}^{ \pm} R_{i}^{\dagger}$ are the rotated spin operators $\left(S_{i}^{ \pm}=S_{i}^{x} \pm \imath S_{i}^{y}\right)$, such that $S_{i}^{+^{\prime}}|\Theta\rangle=0 \forall i$. In the rotated standard basis $\left\{\otimes_{i}\left|k_{i}^{\prime}\right\rangle\right\}\left(S_{i}^{z^{\prime}}\left|k_{i}^{\prime}\right\rangle=\left(S_{i}-k\right)\left|k_{i}^{\prime}\right\rangle\right)$, such that $|\Theta\rangle=\left|0^{\prime}\right\rangle$, and considering first $S_{i}=1 / 2 \forall i$ Eq. (A10) leads, to lowest order in the perturbations (terms quadratic in $\alpha_{i}, \beta_{i j}$ discarded), to a reduced pair state of the form

$$
\rho_{i j} \approx\left(\begin{array}{cccc}
1 & \alpha_{i} & \alpha_{j} & \beta_{i j} \\
\bar{\alpha}_{i} & 0 & 0 & 0 \\
\bar{\alpha}_{j} & 0 & 0 & 0 \\
\bar{\beta}_{i j} & 0 & 0 & 0
\end{array}\right) .
$$

The partial transpose [46,47] of Eq. (A11) has eigenvalues one, zero, and $\pm|\beta|_{i j}$ up to lowest nontrivial order, so that $\rho_{i j}$ will be entangled if $\beta_{i j} \neq 0$. And the exact coefficients $\beta_{i j}$ obtained 
from Eq. (A10) are, for general perturbations $\delta \boldsymbol{h}^{i}$ and $\delta J_{\mu \nu}^{i j}$, not strictly zero for any pair $i, j$ linked by successive applications of the coupling in $H$, due to the two-spin excitations present in the exact eigenstates $|v\rangle$. They can, of course, be very small for distant pairs, but not strictly zero. For higher spins $S, \rho_{i j}$ will be more complex but will still contain a first submatrix of the form of Eq. (A11). Hence, it will also be entangled if $\beta_{i j} \neq 0$, since the partial transpose of this block is the first block of the full partial transpose $\rho_{i j}^{T_{j}}$ and is nonpositive at lowest order, preventing the full $\rho_{i j}^{T_{j}}$ from being positive semidefinite.

For spin $1 / 2$, where the entanglement of formation $E_{i j}$ is just an increasing function of the concurrence $C_{i j}$ [48], Eq. (A11) leads at lowest order to $C_{i j} \approx 2\left|\beta_{i j}\right|$. At this order, $\alpha_{i}, \alpha_{j}$ in Eq. (A11) do not affect $C_{i j}$ nor the eigenvalues of the partial transpose $\rho_{i j}^{T_{j}}$.
[1] M. A. Nielsen and I. Chuang, Quantum Computation and Quantum Information (Cambridge University Press, Cambridge, England, 2000).

[2] S. Haroche and J. M. Raimond, Exploring The Quantum (Oxford University Press, Oxford, 2006).

[3] V. Vedral, Introduction to Quantum Information Science (Oxford University Press, Oxford, 2006).

[4] R. P. Feynman, Opt. News 11, 11 (1985).

[5] D. D. Awschalom, D. Loss, and N. Samarth, Semiconductor Spintronics and Quantum Computing (Springer-Verlag, Berlin, 2002).

[6] D. Loss and D. P. DiVincenzo, Phys. Rev. A 57, 120 (1998).

[7] L. Amico, R. Fazio, A. Osterloh, and V. Vedral, Rev. Mod. Phys. 80, 517 (2008).

[8] N. A. Gershenfeld and I. L. Chuang, Science 275, 350 (1997).

[9] B. E. Kane, Nature (London) 393, 133 (1998).

[10] J. I. Cirac and P. Zoller, Phys. Rev. Lett. 74, 4091 (1995).

[11] A. Shnirman, G. Schön, and Z. Hermon, Phys. Rev. Lett. 79, 2371 (1997).

[12] J. Simon, W. S. Bakr, R. Ma, M. E. Tai, P. M. Preiss, and M. Greiner, Nature (London) 472, 307 (2011).

[13] M. Lewenstein, A. Sanpera, and V. Ahufinger, Ultracold Atoms in Optical Lattices (Oxford University Press, Oxford, 2012).

[14] I. M. Georgescu, S. Ashhab, and F. Nori, Rev. Mod. Phys. 86, 153 (2014).

[15] M. H. Devoret and R. J. Schoelkopf, Science 339, 1169 (2013).

[16] S. Zippilli, M. Grajcar, E. Il'ichev, and F. Illuminati, Phys. Rev. A 91, 022315 (2015).

[17] S. H. W. van der Ploeg, A. Izmalkov, A. M. van den Brink, U. Hübner, M. Grajcar, E. Il'ichev, H.-G. Meyer, and A. M. Zagoskin, Phys. Rev. Lett. 98, 057004 (2007).

[18] R. Barends et al., Nature (London) 534, 222 (2016); R. Barends, J. Kelly, A. Megrant, D. Sank, E. Jeffrey, Y. Chen, Y. Yin, B. Chiaro, J. Mutus, C. Neill, P. O’Malley, P. Roushan, J. Wenner, T. C. White, A. N. Cleland, and J. M. Martinis, Phys. Rev. Lett. 111, 080502 (2013).

[19] D. Porras and J. I. Cirac, Phys. Rev. Lett. 92, 207901 (2004).

[20] K. Kim, M.-S. Chang, R. Islam, S. Korenblit, L.-M. Duan, and C. Monroe, Phys. Rev. Lett. 103, 120502 (2009).

[21] R. Blatt and C. F. Roos, Nat. Phys. 8, 277 (2012).

[22] S. Korenblit et al., New J. Phys. 14, 095024 (2012).

[23] J. W. Britton et al., Nature (London) 484, 489 (2012).

[24] C. Senko, P. Richerme, J. Smith, A. Lee, I. Cohen, A. Retzker, and C. Monroe, Phys. Rev. X 5, 021026 (2015).

[25] J. Kurmann, H. Thomas, and G. Müller, Physica A 112, 235 (1982).

[26] G. Müller and R. E. Shrock, Phys. Rev. B 32, 5845 (1985).
[27] T. Roscilde, P. Verrucchi, A. Fubini, S. Haas, and V. Tognetti, Phys. Rev. Lett. 93, 167203 (2004); T. Roscilde et al., ibid. 94, 147208 (2005).

[28] L. Amico, F. Baroni, A. Fubini, D. Patanè, V. Tognetti, and P. Verrucchi, Phys. Rev. A 74, 022322 (2006); F. Baroni et al., J. Phys. A 40, 9845 (2007).

[29] S. M. Giampaolo, F. Illuminati, P. Verrucchi, and S. De Siena, Phys. Rev. A 77, 012319 (2008).

[30] S. M. Giampaolo, G. Adesso, and F. Illuminati, Phys. Rev. Lett. 100, 197201 (2008); Phys. Rev. B 79, 224434 (2009); Phys. Rev. Lett. 104, 207202 (2010).

[31] R. Rossignoli, N. Canosa, and J. M. Matera, Phys. Rev. A 77, 052322 (2008); 80, 062325 (2009).

[32] N. Canosa, R. Rossignoli, and J. M. Matera, Phys. Rev. B 81, 054415 (2010); L. Ciliberti, R. Rossignoli, and N. Canosa, Phys. Rev. A 82, 042316 (2010).

[33] G. L. Giorgi, Phys. Rev. B 79, 060405(R) (2009); B. Tomasello et al., Europhys. Lett. 96, 27002 (2011).

[34] M. Rezai, A. Langari, and J. Abouie, Phys. Rev. B 81, 060401(R) (2010); J. Abouie, M. Rezai, and A. Langari, Prog. Theor. Phys. 127, 315 (2012).

[35] S. Campbell, J. Richens, N. L. Gullo, and T. Busch, Phys. Rev. A 88, 062305 (2013); G. Karpat, B. Cakmak, and F. F. Fanchini, Phys. Rev. B 90, 104431 (2014).

[36] M. Cerezo, R. Rossignoli, and N. Canosa, Phys. Rev. B 92, 224422 (2015).

[37] L. M. K. Vandersypen and I. L. Chuang, Rev. Mod. Phys. 76, 1037 (2005).

[38] V. Cerletti, W. A. Coish, O. Gywat, and D. Loss, Nanotechnology 16, R27 (2005).

[39] A. Das and B. K. Chakrabarti, Rev. Mod. Phys. 80, 1061 (2008).

[40] M. W. Johnson et al., Nature (London) 473, 194 (2011).

[41] V. Srinivasa, H. Xu, and J. M. Taylor, Phys. Rev. Lett. 114, 226803 (2015).

[42] B. Bertrand, H. Flentje, S. Takada, M. Yamamoto, S. Tarucha, A. Ludwig, A. D. Wieck, C. Bäuerle, and T. Meunier, Phys. Rev. Lett. 115, 096801 (2015).

[43] T. Kontos, M. Aprili, J. Lesueur, F. Genêt, B. Stephanidis, and R. Boursier, Phys. Rev. Lett. 89, 137007 (2002).

[44] C. J. Wellard et al., J. Phys.: Condens. Matter 16, 5697 (2004).

[45] F. Meier, J. Levy, and D. Loss, Phys. Rev. Lett. 90, 047901 (2003).

[46] A. Peres, Phys. Rev. Lett. 77, 1413 (1996).

[47] M. Horodecki, P. Horodecki, and R. Horodecki, Phys. Lett. A 223, 1 (1996).

[48] S. Hill and W. K. Wootters, Phys. Rev. Lett. 78, 5022 (1997); W. K. Wootters, ibid. 80, 2245 (1998). 